\title{
Relaciones entre las desigualdades sociales y la Diabetes Mellitus tipo 2*
}

\section{Relationships between Social Inequalities and Type 2 Diabetes Mellitus}

\section{Relações entre as desigualdades sociais e a Diabete Mellitus tipo 2}

Recepción: 24 de enero de 2018. Aceptación: 16 de octubre de 2019.

Publicación: 30 de junio de 2020.

DOI: https://doi.org/10.11144/Javeriana.rgps19.rdsd

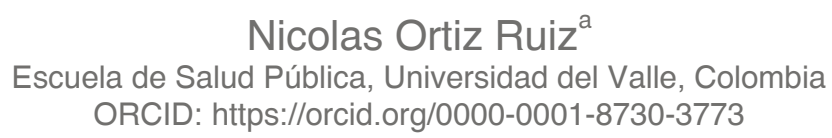

Cómo citar este artículo: Ortiz-Ruiz N. Relaciones entre las desigualdades sociales y la Diabetes Mellitus tipo 2. Revista Gerencia y Políticas de Salud. 2020;19. https://doi.org/10.11144/Javeriana.rgps19.rdsd

a Autor de correspondencia. Correo electrónico: nico6435@yahoo.es 


\section{Resumen}

La diabetes mellitus tipo 2 es una enfermedad de alto impacto en la morbilidad y la mortalidad en el mundo, pues afecta grupos sociales e individuos, según las jerarquías y diferencias sociales. El propósito de este trabajo es identificar las evidencias que muestran relaciones entre desigualdades sociales en los procesos de padecimiento, tratamiento y atención de la diabetes mellitus tipo 2. Para responder al objetivo de este estudio se realizó una revisión integrativa de la literatura, a través de la búsqueda de artículos en revistas indexadas en el período 2016-2018. En general, los resultados mostraron que las desigualdades sociales medidas principalmente por la condición étnico/racial y socioeconómica tiene efectos sobre la incidencia y el tratamiento de la diabetes, en cambio, respecto al género, se observa un comportamiento variable entre hombres y mujeres.

Palabras clave: desigualdades sociales, diabetes mellitus tipo 2, condiciones socioeconómicas, grupos étnicos.

\section{Abstract}

Type 2 diabetes mellitus is a disease with high impact on both the morbidity and mortality worldwide. It affects social groups and individuals with differing social conditions and hierarchies. This work aims to identify the evidence of the relationships between social inequalities regarding the disease process, treatment and health care for type 2 diabetes mellitus. To do so, a comprehensive review of the literature was conducted among indexed journals published during 2016-2018. In general, the results showed that the social inequalities mostly due to the ethnic/race and socioeconomic reasons have effects on the incidence and treatment of type 2 diabetes. On the other hand, regarding the gender there is a variable behavior between men and women.

Keywords: social inequalities, type 2 diabetes mellitus, socioeconomic conditions, ethnic groups.

\section{Resumo}

A diabete mellitus tipo 2 é uma doença de alto impacto na morbimortalidade no mundo, pois afeta grupos sociais e indivíduos, de acordo com hierarquias e diferenças sociais. O objetivo deste trabalho é identificar as evidências que demonstram relações entre desigualdades sociais nos processos de sofrimento, tratamento e atenção ao diabete mellitus tipo 2. Para responder ao objetivo deste estudo realizou-se revisão integrativa da literatura, através da busca de artigos em revistas indexadas no período 2016-2018. Em geral, os resultados mostraram que as desigualdades sociais medidas principalmente pelo status étnico/racial e socioeconômico têm efeitos na incidência e o tratamento do diabete, entanto, em relação ao género, observa-se um comportamento variável entre homens e mulheres.

Palavras-chave: desigualdades sociais, diabete mellitus tipo 2, condições socioeconômicas, grupos étnicos. 


\section{Introducción}

Según estimaciones, en 2014 la prevalencia mundial de diabetes fue de $8.5 \%$ en pacientes mayores de 18 años, lo que equivale a 422 millones de personas (1). En 2012, murieron 1,5 millones de personas como consecuencia directa de la diabetes (1). De esas muertes, $43 \%$ fueron prematuras (antes de los 70 años) y más del $80 \%$ se registraron en países de ingresos bajos y medios (2).

La diabetes mellitus tipo 2 es una enfermedad cuyo desarrollo y evolución está directamente influenciado por las situaciones sociales que afectan a las poblaciones y que particularmente viven los individuos. El comportamiento epidémico de esta enfermedad involucra un complejo grupo de sistemas genéticos y epigenéticos que interactúan dentro de una igualmente compleja estructura social que determina los comportamientos y las prácticas individuales y colectivas (3).

Los estudios coinciden en mostrar que la posición socioeconómica contribuye en el desarrollo de la diabetes a través de procesos complejos que incluyen comportamientos de riesgo, asociados con el tipo de alimentación, la actividad física, el hábito de fumar, el consumo de alcohol y los altos niveles de estrés. Igualmente, con la falta de conocimientos para la prevención y el control adecuado de la enfermedad, la falta de acceso o el acceso desigual a los servicios de salud de calidad $(4,5,6)$.

La diabetes mellitus tipo 2 es un ejemplo típico de enfermedad que parece concentrarse en áreas de mayor pobreza y en individuos con bajo nivel de ingresos y bajo nivel educativo. Esta relación también está condicionada por el papel que tiene la posición socioeconómica en los cuidados de la salud, la prevención de la enfermedad, las medidas de promoción de la salud, la disposición a buscar tratamiento y los estilos de vida (7). La diabetes está asociada a grupos socioeconómicos más desfavorecidos, donde se observa una prevalencia y mortalidad más elevadas en individuos que viven en contextos de privación sociomaterial $(8.9,10,11)$.

En la literatura especializada existe una amplia bibliografía de estudios que analizan las relaciones entre las desigualdades sociales y la diabetes. La presente revisión analiza investigaciones que estudian esas relaciones, partiendo de la inquietud de cómo están siendo estudiadas las desigualdades sociales y la diabetes mellitus tipo 2 y que están mostrando los principales resultados al respecto.

\section{Método}

La técnica utilizada para responder al objetivo de este estudio fue la revisión integrativa de la literatura, que presenta como ventaja la posibilidad de síntesis y de análisis del conocimiento científico producido sobre el tema investigado (12). Esta manera de investigar se caracteriza por presentar un amplio panorama de abordajes metodológicos, que permite la inclusión de múltiples estudios, con diferentes diseños de investigación para la comprensión completa del fenómeno estudiado (12). 
Para esta revisión se siguió un protocolo, con el fin de garantizar el rigor del proceso, que contempla los siguientes componentes: pregunta de revisión, criterios de inclusión y exclusión, estrategias para la búsqueda, selección del material, y análisis y síntesis de la información. La pregunta orientadora fue: ¿cuáles son las evidencias que muestran las relaciones entre desigualdades sociales y el proceso de padecimiento, tratamiento y atención de la diabetes mellitus tipo 2? La base de datos electrónica consultada fue Scopus, durante el mes de septiembre de 2018. Como estrategia de búsqueda, se utilizó la combinación de los descriptores: “inequalities" más “diabetes mellitus tipo 2" y “disparities” más "diabetes mellitus tipo 2".

Se adoptaron como criterios de inclusión: artículos publicados en inglés, español o portugués en el periodo 2016-2018. Entre ellos, artículos y revisiones disponibles en texto completo, publicados en revistas indexadas que en su contenido incluyeran resultados de investigaciones que analizaran cualquier condición social, cultural, política o geográfica que genere estratificación o desventaja social entre individuos o grupos y su relación con el diagnóstico, tratamiento, atención o autogerenciamiento de la diabetes mellitus tipo 2. Se excluyeron estudios teóricos que no presentaran resultados de una investigación, evaluaciones de intervenciones relacionadas con la temática, así como aquellos que no respondieron a los objetivos.

Inicialmente fueron identificados un total de 138 artículos, usando los términos "inequalities" más “diabetes mellitus type 2" y 248 artículos con los términos "disparities" más "diabetes mellitus type 2". En esa primera búsqueda no es estableció ningún periodo de tiempo como filtro. Después, basados en los títulos fueron seleccionados 60 y 58 artículos, respectivamente. Debido a la gran cantidad de artículos, se limitó el periodo de búsqueda al 2016-2018, por lo que la cantidad se redujo a 12 y 40 artículos, de acuerdo con los descriptores de búsqueda. De los 52 artículos totales, fueron eliminados 4, debido a que se repetían. Finalmente, después de leer los resúmenes de los 48 artículos, fueron seleccionados 37.

La Figura 1 muestra el flujograma de las etapas del proceso de selección de los estudios para el análisis, adaptado conforme al protocolo Prisma (13). Para ilustración del lector, de los artículos seleccionados se describe información sobre: autor/año, tipo de estudio, país de realización, local de estudio, variables o categorías para el análisis de las desigualdades sociales y resultados. 


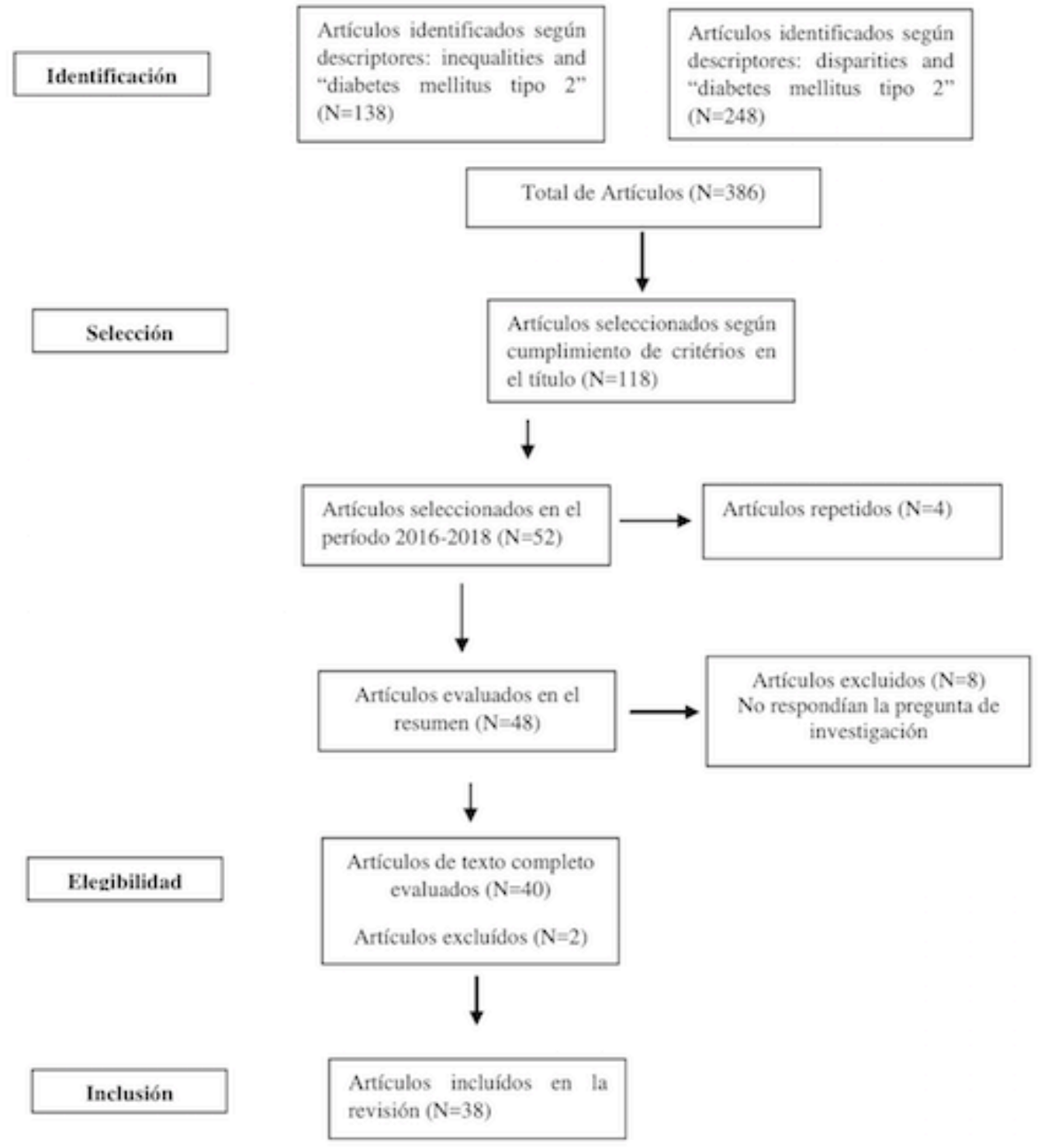

Figura 1. Flujograma del proceso de selección de los artículos Fuente: elaboración propia.

\section{Resultados}

Caracterización de los estudios

La muestra de estudio fue compuesta por 37 artículos, los cuales son presentados en la siguiente tabla, donde se destaca el título/diseño/participantes y objetivos/año. 
Tabla 1. Caracterización de los artículos seleccionados según título, diseño, participantes, objetivo y año de publicación

\begin{tabular}{|c|c|c|c|}
\hline Titulo/diseño/participantes & Objetivos/año & Titulo/diseño/participantes & Objetivos/año \\
\hline $\begin{array}{l}\text { 1. "That wasn't really a place } \\
\text { to worry about diabetes": } \\
\text { Housing access and diabetes } \\
\text { self-management among low- } \\
\text { income adults } \\
\text { Cualitativo/ } 40 \text { participantes }\end{array}$ & $\begin{array}{l}\text { Examinar cómo la } \\
\text { vivienda afecta los } \\
\text { comportamientos } \\
\text { de autogestión de } \\
\text { pacientes con } \\
\text { DMT2. } \\
\text { (2018) }\end{array}$ & $\begin{array}{l}\text { 2. How people from ethnic } \\
\text { minorities describe their } \\
\text { experiences of managing } \\
\text { type-2 diabetes mellitus } \\
\text { Meta-sintesis cualitativa } \\
\text { (27 articulos 1946-2016) }\end{array}$ & $\begin{array}{l}\text { Examinar cómo } \\
\text { las personas de } \\
\text { minorias étnicas } \\
\text { de paises } \\
\text { occidentales } \\
\text { describen sus } \\
\text { experiencias de } \\
\text { manejo de } \\
\text { DMT2. } \\
\quad \text { (2017) }\end{array}$ \\
\hline $\begin{array}{l}\text { 3. Health care experiences of } \\
\text { Indigenous people living with } \\
\text { type } 2 \text { diabetes. } \\
\text { Cualitativo/32 participantes }\end{array}$ & 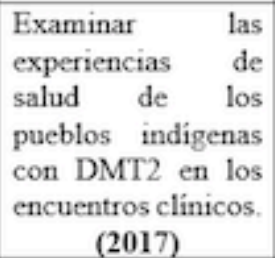 & $\begin{array}{l}\text { 4. U.S. Healthcare } \\
\text { Experiences of Hispanic } \\
\text { Patients with Diabetes and } \\
\text { Family Members. } \\
\text { Cualitativo/172 } \\
\text { participantes }\end{array}$ & \begin{tabular}{lr} 
Explorar las \\
perspectivas de \\
adultos hispanos \\
sobre res \\
experiencias de \\
salud \\
\multicolumn{1}{|}{ (2017) }
\end{tabular} \\
\hline $\begin{array}{l}\text { 5. A Qualitative Study of } \\
\text { Vulnerable Patient Views of } \\
\text { Type } 2 \text { Diabetes Consumer } \\
\text { Reports } \\
\text { Cualitativo/92 participantes }\end{array}$ & $\begin{array}{l}\text { Identificar } \\
\text { poblaciones } \\
\text { vulnerables con } \\
\text { DMT2 perciben los } \\
\text { informes del } \\
\text { consumidor como } \\
\text { útiles para la toma } \\
\text { de decisiones en el } \\
\text { tratamiento de la } \\
\text { DMT2. } \\
\text { (2016) }\end{array}$ & $\begin{array}{l}\text { 6. Type } 2 \text { diabetes mellitus } \\
\text { management among } \\
\text { Ghanaian migrants } \\
\text { resident in three European } \\
\text { countries and their } \\
\text { compatriots in rural and } \\
\text { urban Ghana. The Rodam } \\
\text { study } \\
\text { Transversal/530 } \\
\text { participantes }\end{array}$ & 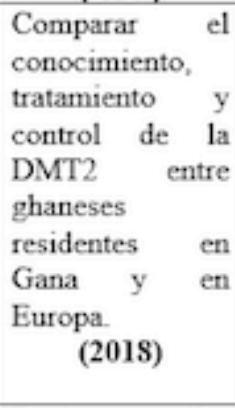 \\
\hline $\begin{array}{l}\text { 7. Self-Care Disparities } \\
\text { Among Adults with Type } 2 \\
\text { Diabetes in the USA } \\
\text { Revision } \\
\text { (25 articulos 2011-2016) }\end{array}$ & 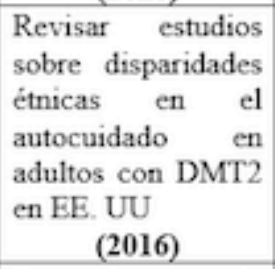 & $\begin{array}{l}\text { 8. Social support and self- } \\
\text { management capabilities in } \\
\text { diabetes patients: An } \\
\text { international observational } \\
\text { study Patient } \\
\text { Transversal/1692 } \\
\text { participantes }\end{array}$ & $\begin{array}{l}\text { Explorar cuáles } \\
\text { aspectos de las } \\
\text { redes sociales se } \\
\text { relacionan con } \\
\text { las capacidades } \\
\text { de autocuidado } \\
\text { (2016) }\end{array}$ \\
\hline $\begin{array}{l}\text { 9. Diabetes Outcome and } \\
\text { Process Measures Among } \\
\text { Patients Who Require } \\
\text { Language Interpreter } \\
\text { Services in Minnesota } \\
\text { Primary Care Practices. } \\
\text { Estudio de cohorte } \\
\text { retrospectivo/13.456 } \\
\text { participantes-Seguimientol } \\
\text { (2012-2013) }\end{array}$ & $\begin{array}{l}\text { Determinar la } \\
\text { adhesión al } \\
\text { tratamiento de } \\
\text { DMT2 y resultados } \\
\text { en pacientes con } \\
\text { limitaciones en el } \\
\text { manejo de inglés en } \\
\text { la atención } \\
\text { primaria. } \\
\text { (2017) }\end{array}$ & $\begin{array}{l}\text { 10. Sex differences in } \\
\text { healthcare expenditures } \\
\text { among adults with } \\
\text { diabetes: evidence from } \\
\text { the medical expenditure } \\
\text { panel survey, 2002-2011 } \\
\text { Transversal/20.442 } \\
\text { participantes }\end{array}$ & $\begin{array}{l}\text { Evaluar las } \\
\text { diferencias en los } \\
\text { gastos indirectos } \\
\text { y totales en salud } \\
\text { en adultos con } \\
\text { DMT2. } \\
\quad \text { (2017) }\end{array}$ \\
\hline
\end{tabular}


Relaciones entre las desigualdades sociales y la Diabetes Mellitus tipo 2

Tabla 1. Caracterización de los artículos seleccionados según título, diseño, participantes, objetivo y año de publicación (continúa)

\begin{tabular}{|c|c|c|c|}
\hline Titulo/diseño/participantes & Objetivos/año & Título/diseño/participantes & Objetivos/año \\
\hline $\begin{array}{l}\text { 11. Insurance-related } \\
\text { disparities in primary care } \\
\text { quality among U.S. Type } 2 \\
\text { diabetes patients } \\
\text { Transversal/componente } \\
\text { doméstico Investigación de } \\
\text { Panel de Gastos Médicos- } \\
2012\end{array}$ & $\begin{array}{l}\text { Explorar las } \\
\text { disparidades } \\
\text { relacionadas al } \\
\text { seguro en la calidad } \\
\text { de la atención } \\
\text { primaria } \\
\text { americanos en } \\
\text { DMT2. con } \\
\text { (2016) }\end{array}$ & $\begin{array}{l}\text { 12. Variation in the use of } \\
\text { primary care services for } \\
\text { diabetes management } \\
\text { according to country of } \\
\text { birth and geography } \\
\text { among older Australians } \\
\text { Transversal/ } \mathbf{8 4 0} \\
\text { participantes }\end{array}$ & 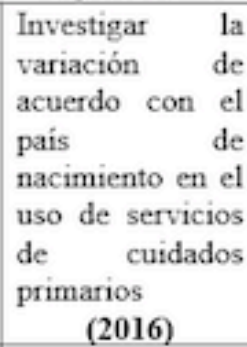 \\
\hline $\begin{array}{l}\text { 13. Cumulative social risk and } \\
\text { type } 2 \text { diabetes in U.S. adults: } \\
\text { The National Health and } \\
\text { Nutrition Examination } \\
\text { Survey (Nhanes) 1999-2006 } \\
\text { Transversal/10.276 } \\
\text { participantes }\end{array}$ & $\begin{array}{l}\text { Medir los efectos } \\
\text { acumulativos de } \\
\text { factores sociales } \\
\text { adversos sobre el } \\
\text { riesgo de la DMT2. } \\
\text { (2016) }\end{array}$ & $\begin{array}{l}\text { 14. The association of early } \\
\text { life socioeconomic } \\
\text { conditions with } \\
\text { prediabetes and type } 2 \\
\text { diabetes: results from the } \\
\text { Maastricht study } \\
\text { Transversal } / 3263 \\
\text { participantes }\end{array}$ & $\begin{array}{l}\text { Examinar la } \\
\text { asociación de las } \\
\text { condiciones } \\
\text { socioeconómicas } \\
\text { en el inicio de la } \\
\text { vida con } \\
\text { prediabetes y } \\
\text { DM2 en la edad } \\
\text { adulta. } \\
\text { (2017) }\end{array}$ \\
\hline $\begin{array}{l}\text { 15. Socioeconomic status and } \\
\text { prevalence of type } 2 \text { diabetes } \\
\text { in mainland China, Hong } \\
\text { Kong and Taiwan: a } \\
\text { systematic review } \\
\text { Revisión sistemática (33 } \\
\text { articulos 1946-2016/1973- } \\
2016 / 1990-2016)\end{array}$ & $\begin{array}{l}\text { Describir la } \\
\text { asociación entre el } \\
\text { estatus } \\
\text { socioeconómico y } \\
\text { la prevalencia de } \\
\text { DMT2 en chinos. } \\
\text { (2017) }\end{array}$ & $\begin{array}{l}\text { 16. Social disparities in } \\
\text { diabetes care: a general } \\
\text { population study in } \\
\text { Denmark. } \\
\text { Transversal/907 } \\
\text { participantes }\end{array}$ & $\begin{array}{l}\text { Investigar la } \\
\text { asociación entre } \\
\text { factores } \\
\text { socioeconómicos } \\
\text { y la consecución } \\
\text { de objetivos de } \\
\text { tratamiento en } \\
\text { pacientes con } \\
\text { DMT2. } \\
\text { (2017) }\end{array}$ \\
\hline $\begin{array}{l}\text { 17. Socioeconomic disparities } \\
\text { in type } 2 \text { diabetes mellitus } \\
\text { prevalence and self- } \\
\text { management behaviors in } \\
\text { rural southwest China } \\
\text { Transversal/5532 } \\
\text { participantes }\end{array}$ & $\begin{array}{l}\text { Examinar la } \\
\text { asociación entre la } \\
\text { prevalencia y } \\
\text { autogerenciamiento } \\
\text { de la DMT2 entre } \\
\text { grupos étnicos } \\
\qquad(\mathbf{2 0 1 6 )}\end{array}$ & $\begin{array}{l}\text { 18. Health inequalities in } \\
\text { diabetes mellitus and } \\
\text { hypertension: A parish } \\
\text { level study in the northern } \\
\text { region of Portugal } \\
\text { Transversal/2025 } \\
\text { participantes }\end{array}$ & $\begin{array}{l}\text { Medir la } \\
\text { distribución } \\
\text { geográfica y } \\
\text { socioeconómica } \\
\text { de DMT2 e } \\
\text { hipertensión } \\
\text { arterial en el } \\
\text { norte de } \\
\text { Portugal. } \\
\quad(2016)\end{array}$ \\
\hline $\begin{array}{l}\text { 19. Long-term effects of } \\
\text { neighbourhood deprivation } \\
\text { on diabetes risk: Quasi- } \\
\text { experimental evidence from } \\
\text { a refugee dispersal policy in } \\
\text { Sweden. }\end{array}$ & $\begin{array}{l}\text { de las condiciones } \\
\text { del lugar de } \\
\text { vivienda en el } \\
\text { riesgo de DMT2 de }\end{array}$ & $\begin{array}{l}\text { 20. The prevalence of } \\
\text { diabetes mellitus and its } \\
\text { associated factors in the } \\
\text { Brazilian adult population: } \\
\text { Evidence from a } \\
\text { population-based survey }\end{array}$ & $\begin{array}{lr}\text { Estimar } & \text { la } \\
\text { prevalencia } & \text { de } \\
\text { DMT2 y } & \text { sus } \\
\text { factores } & \\
\text { asociados } & \text { en }\end{array}$ \\
\hline
\end{tabular}


Tabla 1. Caracterización de los artículos seleccionados según título, diseño, participantes, objetivo y año de publicación (continúa)

\begin{tabular}{|c|c|c|c|}
\hline Titulo/diseño/participantes & Objetivos/año & Titulo/diseño/participantes & Objetivos/año \\
\hline $\begin{array}{c}\text { Transversal/61.386 } \\
\text { participantes }\end{array}$ & $\begin{array}{l}\text { refugiados en } \\
\text { Suecia. } \\
\qquad(2016)\end{array}$ & $\begin{array}{c}\text { Transversal/12.423 } \\
\text { participantes }\end{array}$ & $\begin{array}{l}\text { población adulta } \\
\text { brasilera. } \\
(2017)\end{array}$ \\
\hline $\begin{array}{l}\text { 21. Socioeconomic status and } \\
\text { prevalence of self-reported } \\
\text { diabetes among adults in } \\
\text { Tehran: Results from a large } \\
\text { population-based cross- } \\
\text { sectional study (Urban Heart- } \\
\text { 2) Transversa/91.s14 } \\
\text { participantes }\end{array}$ & 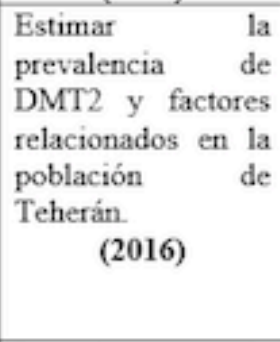 & $\begin{array}{l}\text { 22. Education achievement } \\
\text { and type } 2 \text { diabetes-What } \\
\text { mediates the relationship } \\
\text { in older adults? } \\
\text { Estudio de cohorte } 7462 \\
\text { participantes-Seguimiento } \\
\text { (2000-02-2010) }\end{array}$ & $\begin{array}{l}\text { Identificar } \\
\text { factores } \\
\text { mediadores en el } \\
\text { éxito escolar y la } \\
\text { DMT2 incidente } \\
\text { en adultos } \\
\text { mayores } \\
\text { (2017) }\end{array}$ \\
\hline $\begin{array}{l}\text { 23. Educational level is related } \\
\text { to physical fitness in patients } \\
\text { with type } 2 \text { diabetes - A } \\
\text { cross-sectional study (2016) } \\
\text { Transversal/185 } \\
\text { participantes }\end{array}$ & $\begin{array}{l}\text { Investigar la } \\
\text { relación entre el } \\
\text { nivel educativo, el } \\
\text { condicionamento } \\
\text { aeróbico, fuerza } \\
\text { muscular funcional } \\
\text { de miembros } \\
\text { inferiores y DMT2 } \\
\text { (2016) }\end{array}$ & $\begin{array}{l}\text { 24. Gender differences in } \\
\text { cumulative life-course } \\
\text { socioeconomic position } \\
\text { and social mobility in } \\
\text { relation to new onset } \\
\text { diabetes in adults } \\
\text { Transversal/ } 15.105 \\
\text { participantes }\end{array}$ & $\begin{array}{l}\text { Investigar la } \\
\text { asociación de la } \\
\text { posición } \\
\text { socioeconómica } \\
\text { acumulativa y la } \\
\text { movilidad social } \\
\text { con el inicio de } \\
\text { DMT2 en } \\
\text { adultos en Brasil } \\
\text { (2016) }\end{array}$ \\
\hline $\begin{array}{l}\text { 25. Association of modifiable } \\
\text { risk factors in young } \\
\text { adulthood with racial } \\
\text { disparity in incident type } 2 \\
\text { diabetes during middle } \\
\text { adulthood } \\
\text { Estudio de cohorte/4251 } \\
\text { participantes-seguimiento } \\
\text { (1985/1986-2015/2016). }\end{array}$ & $\begin{array}{l}\text { Determinar las } \\
\text { asociaciones de } \\
\text { factores biológicos, } \\
\text { de vecindad, } \\
\text { psicosociales, } \\
\text { socioeconómicos y } \\
\text { comportamentales } \\
\text { en la edad adulta } \\
\text { joven con la } \\
\text { disparidad racial } \\
\text { observada en la } \\
\text { incidencia de } \\
\text { DMT2. } \\
\text { (2017) }\end{array}$ & $\begin{array}{l}\text { 26. Racial and Ethnic } \\
\text { Disparities in Diabetes } \\
\text { Screening Between Asian } \\
\text { Americans and Other } \\
\text { Adults: BRFSS 2012- } \\
\text { 2014. } \\
\text { Transversal/526.000 } \\
\text { participantes }\end{array}$ & $\begin{array}{l}\text { Evaluar las } \\
\text { disparidades } \\
\text { raciales y étnicas } \\
\text { en la atención de } \\
\text { la DMT2 en } \\
\text { americanos } \\
\text { asiáticos y otros } \\
\text { adultos. } \\
\text { (2017) }\end{array}$ \\
\hline $\begin{array}{l}\text { 27. The diabetes disparity and } \\
\text { puerto rican identified } \\
\text { individuals: A systematic } \\
\text { literature review } \\
\text { Revisión sistemática } \\
(7 \text { articulos-2007-2017) }\end{array}$ & $\begin{array}{l}\text { Describir a } \\
\text { disparidad de la } \\
\text { DMT2 en adultos } \\
\text { de Puerto Rico que } \\
\text { viven en Estados } \\
\text { Unidos. } \\
\text { (2017) }\end{array}$ & $\begin{array}{l}\text { 28. Racial/Ethnic } \\
\text { Residential Segregation, } \\
\text { Obesity, and Diabetes } \\
\text { Mellitus } \\
\text { Revisión comprensiva } \\
\text { (14 articulos-2013-2015) }\end{array}$ & $\begin{array}{l}\text { Revisar los } \\
\text { resultados de } \\
\text { estudios sobre } \\
\text { segregación } \\
\text { residencial, } \\
\text { racial/étnica con } \\
\text { la obesidad y la } \\
\text { DMT2 } \\
\quad(2016)\end{array}$ \\
\hline
\end{tabular}


Relaciones entre las desigualdades sociales y la Diabetes Mellitus tipo 2

Tabla 1. Caracterización de los artículos seleccionados según título, diseño, participantes, objetivo y año de publicación (continúa)

\begin{tabular}{|c|c|c|c|}
\hline Titulo/diseño/participantes & Objetivos/año & Titulo/diseño/participantes & Objetivos/año \\
\hline $\begin{array}{l}\text { 29. Healthcare mistreatment } \\
\text { attributed to discrimination } \\
\text { among mapuche patients and } \\
\text { discontinuation of diabetes } \\
\text { care } \\
\text { Transversal/85 participantes }\end{array}$ & \begin{tabular}{lr} 
Examinar & los \\
efectos del maltrato & la \\
sobre & del \\
continuidad & de \\
tratamiento & DMT2 en pacientes \\
\multicolumn{2}{c}{ mapuches de Chile. } \\
\multicolumn{2}{c}{ (2016) }
\end{tabular} & $\begin{array}{l}\text { 30. Ethnic difference in the } \\
\text { prevalence of pre-diabetes } \\
\text { and diabetes mellitus in } \\
\text { regions with sami and non- } \\
\text { sami populations in } \\
\text { Norway - The Saminor } 1 \\
\text { study } \\
\text { Transversal/15.208 } \\
\text { participantes }\end{array}$ & 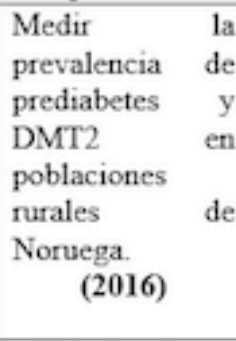 \\
\hline Titulo/diseño/participantes & Objetivos/año & Titulo/diseño/participantes & Objetivos/año \\
\hline $\begin{array}{l}\text { 31. Stress and other } \\
\text { determinants of diabetes- } \\
\text { specific quality of life in low- } \\
\text { income african americans } \\
\text { with uncontrolled type } 2 \\
\text { diabetes mellitus } \\
\text { Transversal/211participantes }\end{array}$ & \begin{tabular}{l} 
Comprender las \\
relaciones de la \\
calidad de vida por \\
DMT2 y datos \\
demografico, \\
clinicas y \\
sicosociales en \\
afroamericanos. \\
\multicolumn{2}{|c|}{$(2016)$}
\end{tabular} & $\begin{array}{l}\text { 32. Relative contributions of } \\
\text { socioeconomic, local } \\
\text { environmental, } \\
\text { psychosocial, } \\
\text { lifestyle/behavioral, } \\
\text { biophysiological, and } \\
\text { ancestral factors to } \\
\text { racial/ethnic disparities in } \\
\text { type } 2 \text { diabetes } \\
\text { Transversal/2764 } \\
\text { participantes }\end{array}$ & $\begin{array}{l}\text { Evaluar las } \\
\text { contribuciones } \\
\text { relativas de seis } \\
\text { dominios de } \\
\text { influencia con } \\
\text { las disparidades } \\
\text { raciales/étnicas } \\
\text { en la DMT2. } \\
\text { (2016) }\end{array}$ \\
\hline $\begin{array}{l}\text { 33. Disparities in type } 2 \\
\text { diabetes prevalence among } \\
\text { ethnic minority groups } \\
\text { resident in Europe } \\
\text { Revisión sistemática- } \\
\text { metaanálisis/20 articulos } \\
\text { (1994-2014) }\end{array}$ & $\begin{array}{l}\text { Cuantificar la } \\
\text { variación en el } \\
\text { riesgo de DMT2 } \\
\text { entre población de } \\
\text { fuera de Europa y } \\
\text { población europea. } \\
\text { (2016) }\end{array}$ & $\begin{array}{l}\text { 34. Contributors to Patients' } \\
\text { Ratings of Quality of Care } \\
\text { Among Ethnically Diverse } \\
\text { Patients with Type } 2 \\
\text { Diabetes } \\
\text { Transversal/1320 } \\
\text { participantes }\end{array}$ & $\begin{array}{l}\text { Explorar la } \\
\text { relación médico- } \\
\text { paciente en } \\
\text { americanos } \\
\text { vietnamitas, } \\
\text { mexicanos } \\
\text { blancos no- } \\
\text { hispanos con } \\
\text { DMT2. } \\
\quad \text { (2016) }\end{array}$ \\
\hline $\begin{array}{l}\text { 35. Racial-ethnic disparities in } \\
\text { the association between risk } \\
\text { factors and diabetes: The } \\
\text { Northern Manhattan Study } \\
\text { Estudio de cohorte/2430 } \\
\text { participantes-seguimiento } \\
\text { (1993-2014) }\end{array}$ & $\begin{array}{l}\text { Identificar factores } \\
\text { de riesgo de DMT2 } \\
\text { y los efectos de la } \\
\text { raza-etnia } \\
\text { (2016) }\end{array}$ & $\begin{array}{l}\text { 36. Culture and risk } \\
\text { assessments: Why latino } \\
\text { americans perceive greater } \\
\text { risk for diabetes } \\
\text { Transversal/tres estudios } \\
\text { independientes }\end{array}$ & \begin{tabular}{l} 
Examinar \\
diferencias en \\
vulnerabilidad \\
percibida en \\
americanos \\
europeos y \\
latinos. \\
\multicolumn{1}{|c|}{ (2016) }
\end{tabular} \\
\hline $\begin{array}{l}\text { 37. Diabetes self-management } \\
\text { behaviors, medical care, } \\
\text { glycemic control, and self- } \\
\text { rated health in U.S. men by } \\
\text { race/ethnicity }\end{array}$ & $\begin{array}{l}\text { Examinar } \\
\text { diferencias étnicas } \\
\text { en hombres con } \\
\text { DMT2 en el } \\
\text { cuidado médico, } \\
\text { control de glicemia }\end{array}$ & $\begin{array}{l}\text { 38. Racial/ethnic disparities } \\
\text { in primary care quality } \\
\text { among type } 2 \text { diabetes } \\
\text { patients, } \\
\text { expenditure panel survey, } \\
2012\end{array}$ & $\begin{array}{l}\text { Explorar } \\
\text { disparidades } \\
\text { étnicas en la } \\
\text { calidad de la } \\
\text { atención } \\
\text { primaria }\end{array}$ \\
\hline $\begin{array}{c}\text { Transversal/646 } \\
\text { participantes }\end{array}$ & $\begin{array}{l}\text { y autoevaluación de } \\
\text { salud } \\
\qquad(2016)\end{array}$ & $\begin{array}{c}\text { Transversal/2.617 } \\
\text { participantes }\end{array}$ & $\begin{array}{l}\text { americanos con } \\
\text { DMT2. } \\
\text { (2016) }\end{array}$ \\
\hline
\end{tabular}

Fuente: elaboración propia. 
Según el lugar donde se llevó a cabo la investigación, se encontró que diecinueve (19) corresponden a investigaciones realizadas en los Estados Unidos (1, 4, 5, 7, 9,10,11, 13, $14,15,16,17,18,19,20,21,22,23,24)$, once (11) en países de Europa (Holanda, Alemania, Suecia, Suiza, Portugal, Dinamarca y Noruega) (2, 6, 8, 25,26,27,28,29,30,31,32), y tres (3) en países de América Latina (Brasil e Chile) $(20,24,29)$. Los cuatro estudios restantes fueron adelantados en China (2), Irán (1) y Canadá (1) $(3,12,33,34,35)$.

De los 37 artículos, 5 usaron abordajes cualitativos $(1,2,3,4,5)$ y 32, cuantitativos $(6,38)$. Dentro de los diseños cualitativos, 4 de ellos utilizan fuentes primarias y uno fue una revisión bibliográfica (meta-síntesis). Los diseños cuantitativos incluyen cinco (5) revisiones (tres revisiones sistemáticas, una revisión sistemática/metaanálisis y una revisión comprensiva) (33, $16,17,32)$, y 27 fueron investigaciones que usaron otros métodos basados en análisis de fuentes primarias $(6,8,26,34,35,30,14,38,18,20,22)$ y secundarias $(9,10,11,13,27,28,29,36,37$, $15,31,19,21,23,24,25)$.

De las investigaciones cualitativas, dos analizaron desigualdades relacionadas con el autogerenciamiento, y las otras tres, con los servicios y las experiencias de atención. Las cuatro investigaciones que consultaron fuentes primarias hicieron entrevistas individuales semiestructuradas y grupos focales. En todos los casos se realizó análisis temático, esto es, codificación a partir de la identificación de temas recurrentes, categorización y análisis interno entre las categorías. La metasíntesis incluyó estudios cualitativos, realizados entre 1946 y 2016 , que presentasen resultados de adultos de minorías étnicas.

Con relación a las variables o categorías asociadas con las desigualdades sociales, cuatro (4) de los artículos usaron categorías étnicas: minorías étnicas (indígenas, hispanos y afroamericanos). En esos estudios, adicionalmente se incluyen variables territoriales (urbano-rural) y de género. El otro artículo empleó las condiciones de acceso a vivienda en población de bajos ingresos. En ese estudio se usaron variables de clasificación de los participantes, como grupo étnico, género, subsidio de vivienda, habitación de calle, idioma primario, tipo de seguro de salud y fuente primaria de ingresos.

De los 32 artículos que usaron abordajes cuantitativos, diecinueve (19) se enfocaron en el análisis de las condiciones étnicas relacionadas con la diabetes. De estos, doce (12) fueron estudios transversales $(6,28,15,38,31,18,19,20$, 22,23,24); cuatro (4), revisiones (7, 16, 17, 32); y tres, estudios de cohorte (3) $(9,14,21)$. En relación con la condición étnico/racial, los estudios incluyeron la pertenencia o identificación de los participantes en minorías 
étnicas, como migrantes, refugiados, hispanos/latinos, afroamericanos, negros no hispanos, asiáticoamericanos, indios no hispanos, nativos de Alaska, multirraciales y no hispanos. Algunos de los estudios incluyeron las categorías de blancos o blancos europeos. También usaron otras variables relacionadas con las desigualdades sociales, como segregación, condiciones de vivienda, género, edad, nivel educativo, ingresos, tipo de seguro en salud y situación laboral (empleado/desempleado).

Los quince (15) artículos restantes se enfocaron principalmente en condiciones socioeconómicas. Trece (13) de ellos fueron estudios transversales $(8,10,11,13,25,26,27$, $33,34,35,36,37,30)$, y se encontró un (1) estudio de cohorte (29) y una (1) revisión sistemática (33). Las variables usadas para medir las condiciones socioeconómicas fueron las "condiciones socioeconómicas iniciales", medidas a través de la pobreza en la juventud y el nivel educativo de los padres. Las "condiciones socioeconómicas en la edad adulta", medidas por el nivel de ingresos y educación del participante. Se usó, también, el estatus socioeconómico (ESE), medido por el nivel de educación, ingresos y ocupación.

Igualmente, fue utilizado el índice de posición socioeconómica (PSE), que incluye activos domésticos (ingresos familiares anuales y equipamiento del hogar), nivel educativo y acceso a servicios médicos. También, PSE del curso de la vida, el cual incluye el nivel de educación y la clase social ocupacional a lo largo de la vida. Otro de los estudios usó la ocupación, a través de la Clasificación Internacional Estándar de Ocupaciones del 2008 y, para medir el estatus socioeconómico, empleó el índice de riqueza que incluye activos inmobiliarios, propiedad y área de la casa.

\section{Principales hallazgos}

\section{Abordajes cualitativos}

En todos los estudios se encontraron evidencias que muestran relaciones entre desigualdades económicas, sociales y culturales con el autogerenciamiento de la diabetes y experiencias de atención en salud. La falta de acceso y la instabilidad de la vivienda afectó la capacidad de los participantes para: 1) priorizar el tratamiento de la diabetes, 2) establecer y mantener rutinas de autogerenciamiento de la diabetes y 3 ) cubrir gastos relacionados con la diabetes (1).

En la metasíntesis se encontró que en las personas de minorías étnicas, el autogerenciamiento estuvo condicionado por la sensación de impotencia, cuestiones de accesibilidad y aceptabilidad del tratamiento, así como por los roles culturalmente definidos dentro de las familias. También se encontraron relaciones con el significado cultural de los alimentos y el efecto cultural del 
estigma (2). Al respecto de las experiencias de atención, en la población indígena de Canadá se perciben rastros del legado colonial en los servicios de salud. Existe resistencia de la población indígena a ser tratada por el sistema occidental, porque percibe imposiciones en algunos tratamientos que recuerdan relaciones autoritarias históricas. Percibe que los cuidados de salud refuerzan relaciones históricas de racismo, discriminación y estereotipos, expresados en la negación o entrega de cuidados de menor calidad y en las políticas que no apoyan las prácticas culturales para el cuidado (3). Identificaron, también, barreras estructurales en torno al acceso a los servicios, la falta de médicos, aislamiento geográfico, rotación del personal de salud y falta de continuidad de los cuidados. Identifican problemas en la comunicación entre médico y paciente, como un aspecto que afecta el cuidado y la mitigación de daños. Se señala que los profesionales se enfocan en dar medicamentos y tratar lo que está mal, pero no escuchan a los pacientes, tampoco explican adecuadamente ni acuerdan con el paciente su propio tratamiento.

En los estudios que analizaron la atención, los pacientes perciben discriminación e interacciones deficientes con los proveedores. Reclaman información suficiente y clara, relaciones atentas y respetuosas y, en general, una mejor atención (4). En la misma línea, otros resultados indican poco interés de parte de los pacientes en los datos comparativos sobre el desempeño del proveedor de los servicios. En vez de eso, desatacaron como prioritaria la calidad de las interacciones interpersonales y las referencias personales para escoger su médico. También, están interesados en información más básica, simple y específica para ayudar en el automanejo de la diabetes (5).

\section{Abordajes cuantitativos}

\section{Condiciones étnico/raciales y diabetes}

Por otro lado, se observó mayor prevalencia en las minorías étnicas estudiadas: la población Sami en comparación con no Sami en Noruega (prevalencia de diabetes mellitus en hombres Sami fue de 5,5\%; y en mujeres, de 4,8\%, mientras que en hombres no Sami fue de 4,6\%; y en mujeres, de 4,5\%) (38). El riesgo de diabetes mellitus tipo 2 en grupos étnicos minoritarios que viven en Europa es tres a cinco veces mayor entre las personas del Sur de Asia, dos a cuatro veces mayor en personas del Norte de África y dos a tres veces mayor entre las personas de África Subsahariana (19). Por otra parte, en un grupo de migrantes/refugiados en Suecia, se observó un aumento de las probabilidades de diagnóstico de diabetes de $15 \%$ y 22\%, al comparar áreas de moderada y alta privación con áreas de baja privación (27).

En la misma dirección, se identificó mayor prevalencia de diabetes en población negra e hispana en comparación con la blanca, mediada principalmente por el riesgo socioeconómico (18). En una cohorte, se observó mayor riesgo de tener diabetes en población negra no hispana (HR 1.69) e hispana (HR 2.25), en comparación con el grupo de blancos no hispanos. En contraste, una revisión no encontró asociación entre la segregación residencial étnicoracial y la prevalencia 
de diabetes, no obstante, mayor segregación de población negra fue relacionada con mayor mortalidad por diabetes (16).

Relacionando las condiciones étnicas con el acceso a los servicios y el autogerenciamiento, estudios muestran que los asiáticos americanos tuvieron 34\% menos chance de recibir una prueba de diabetes recomendada (prueba para azúcar elevada en sangre o diabetes en los últimos 3 años), en comparación con los blancos no hispanos (14). Otros resultados indican que los puertorriqueños que viven en los Estados Unidos tienden a tener mayores facilidades que otros hispanos para acceder a los servicios por el estatus de ciudadanía y el manejo del inglés (15). Con algunas diferencias con lo presentado anteriormente, se encontró que los negros no hispanos tuvieron mayores chances de consulta de un especialista en diabetes $(84,9 \%)$, seguidos por blancos no hispanos $(74,7 \%)$, mientras que los hispanos reportaron las proporciones más bajas (55,2\% americanos mexicanos, y $62,1 \%$ otros hispanos). Sin embargo, resultados sobre el autogerenciamiento indican que los blancos no hispanos tuvieron la menor proporción de adhesión al consumo de grasas saturadas $(<10 \%$ de calorías de grasas saturadas) y una mayor proporción de reportes de actividad física en el trabajo y en tiempos de ocio (20).

Los pacientes con manejo limitado del inglés en los Estados Unidos, que requieren servicios de intérprete, fueron más propensos a atender las recomendaciones de control de la presión arterial (OR 2,02); examen de hemoglobina medida en los 6 meses anteriores (OR 1,23), y de colesterol LDL, medido en los últimos 12 meses (OR 1,4) (9). Otro estudio que analizó las prácticas de autocuidado en grupos étnicos en los Estados Unidos encontró que los negros e hispanos tienen menores niveles de adhesión a los medicamentos que los blancos. En sentido contrario, los hispanos tienen una dieta más saludable que los blancos. También, las personas negras no hispanas mostraron estar más comprometidas con el automonitoreo regular de la glucosa en sangre que los blancos (7).

Respecto a la calidad de la atención primaria en minorías étnicas y población blanca, usando medidas de primer contacto, longitudinalidad, integralidad y coordinación, se encontraron tasas similares en todas las categorías étnicas de acuerdo (23). Otros análisis que comparan el uso de servicios de población de Gana, que vive en Gana y en Europa, encontraron que la sensibilización sobre la diabetes fue menor en la zona rural de Gana (51\%), que en los países de Europa, los cuales varían de 73\% en Londres (OR 2,7) a 79\% en Ámsterdam (OR 4,7). El tratamiento también fue más bajo en la zona rural (37\%) que en la urbana de Gana (56\%; OR $2,6)$ y en los países europeos $(67 \%$ en Londres, OR 3,4, comparado con $73 \%$ en Berlín, OR $6,9)$. En contraste, el control de la diabetes en la zona rural de Gana (63\%) fue comparable con el Ámsterdam y Berlín, pero mayor que el de Londres $(40 \%$; OR 0,4) y Gana urbana $(28 \%$; OR 0,3) (6).

Al analizar las relaciones de pacientes de diferentes grupos étnicos que viven en Estados Unidos, con el personal médico se encontró que los vietnamitas, en comparación con los blancos no hispanos y los mexicanos-americanos, calificaron más bajo el nivel de participación en la toma de decisiones relacionadas con su tratamiento $(50,9,52,1$ y $52,6, \mathrm{p}=0.01)$. Sin embargo, los 
pacientes blancos no hispanos calificaron más bajo la confianza en su proveedor de salud en comparación con los mexicanos y los vietnamitas $(84,87,9$ e 88,5, p =0.01) (32).

En un estudio con población indígena Mapuche de Chile, se reporta que el evento clínico negativo más frecuente fue que el profesional realizaba el examen físico apresuradamente (41\%), seguido por la entrega de información insuficiente (35\%) y la falta de explicación de los resultados de los exámenes (32\%). Los pacientes atribuyen las experiencias negativas en salud a los preconceptos sobre su etnia y su nivel socioeconómico, situación que conlleva a interrumpir el tratamiento, lo que lleva a aplazar la siguiente consulta o a cambiar el profesional con quien se tuvo una experiencia negativa (17).

\section{Condiciones socioeconómicas y diabetes}

En los países bajos se encontró que las personas con bajo nivel socioeconómico en el inicio de sus vidas presentaron una probabilidad 1,56 veces mayor de prediabetes y 1,61 mayor de tener diabetes (13). En el mismo sentido, en China, Hong Kong y Taiwán, la baja escolaridad está asociada a un aumento de la prevalencia de diabetes tipo 2 (OR varía de 0,39 a 1,52 para el más alto, en comparación con el menor nivel de escolaridad), mientras que la asociación entre ingresos, ocupación y la diabetes tipo 2 no es clara. Solo un pequeño número de estudios identificó una asociación significativa entre ocupación y diabetes tipo 2 (25).

En Dinamarca se encontró que el bajo nivel de escolaridad (educación básica) y los bajos ingresos familiares (menos de $21.400 €$ por año) mostraron asociación con la no obtención de metas en el tratamiento de diabetes. Pacientes con bajo nivel socioeconómico eran más frecuentemente obesos, físicamente inactivos, fumadores y tenían presión arterial elevada (33). En China, se reportó que el nivel educativo individual fue negativamente asociado con la prevalencia de diabetes, mientras que los individuos con mayor patrimonio familiar y mayor PSE fueron más propensos a padecer de diabetes. Sin embargo, las personas con diabetes, con mayor patrimonio familiar, mayor nivel de escolaridad y mayor PSE presentaron mayor probabilidad de automonitoreo de glicemia, de adhesión a los medicamentos prescritos y a tomar medidas para controlar la diabetes (26).

En Portugal se encontró asociación entre la baja escolaridad, desempleo y bajos ingresos. Las diferencias de prevalencia de diabetes entre los deciles más y los menos favorecidos, fueron de 1,31\% (ingresos), 1,17\% (educación superior), 0,79\% (desempleo), 0,62\% (analfabetismo), $0,55 \%$ (condiciones de vivienda) y $0,39 \%$ (acceso a servicios de salud) (34). En la misma dirección, en Teherán la prevalencia de la diabetes fue significativamente menor en quienes tienen mayor puntuación de riqueza, mayor educación y viven en áreas más acomodadas (36).

Una investigación en los Estados Unidos analizó los efectos acumulativos de factores sociales adversos sobre el riesgo de desarrollar diabetes, donde encontró que al comparar un puntaje de 
riesgo social acumulativo de $0 \mathrm{y} \geq 3$, el chance de diabetes ajustado por edad y sexo fue de 2,84 e 2,72, respectivamente (riesgo social: bajos ingresos familiares, baja escolaridad, estatus de grupo étnico/racial minoritario y estatus de vivienda) (12).

En Brasil, un estudio indica que después del ajuste, la diabetes permaneció asociada a la edad ( $\geq 40$ años), escolaridad ( $<8$ años de estudio), estado civil (soltero), obesidad, sedentarismo, comorbilidad con hipertensión e hipercolesterolemia. No se encontró asociación entre clase económica y diabetes (28). En Alemania, se encontró en una cohorte que el riesgo de incidencia de diabetes disminuyó conforme aumenta el nivel de educación (HR educación superior: 0,52; enseñanza media: 0,8$)(35)$.

Igualmente, con mayores niveles de escolaridad aumentó la participación en actividades físicas, al menos una vez por semana, y el consumo de frutas, legumbres y suplementos vitamínicos diariamente. Aquellos de menor nivel educativo fueron más propensos a tener mayor índice de masa corporal, proteína C-reactiva (PCR) y triglicéridos en ayunas (35). En otra experiencia en Suiza, se observó que, en general, el condicionamiento aeróbico fue $46 \%$, la fuerza muscular de un miembro funcional, 16\%; y la velocidad de marcha, 11\% mayor en pacientes con niveles educativos altos (29).

En Estados Unidos un estudio analizó el estatus del seguro de salud y los atributos de la atención primaria relacionadas con el primer contacto, donde se reveló que, en términos de longitudinalidad, integralidad y coordinación, no hubo diferencias estadísticamente significativas entre tipos de seguro y calidad de la atención primaria. Se afirma que los factores de estratificación socioeconómica (educación, situación de empleo e ingresos) son considerados las causas de las disparidades en los cuidados (11).

En Brasil, al analizar nuevos casos de diabetes por género, a partir de las condiciones acumulativas en las posiciones socioeconómicas en el curso de la vida, se encontró que aumentaron los nuevos casos con el incremento de las desventajas socioeconómicas en el curso da vida, principalmente en los hombres. Esta diferencia por género fue pronunciada en cuanto los procesos acumulativos fueron evaluados por los puntajes basados en la educación (alta versus baja desventaja social acumulativa, OR 4,7; en hombres, OR 2 en mujeres) (30). En cuanto a los gastos para el tratamiento, un estudio en Estados Unidos arroja que las mujeres con diabetes tuvieron estadísticamente mayores gastos de bolsillo (out of pocket, OOP) y gastos directos totales, en comparación con los hombres. Tienen \$US 218 más gastos de bolsillo al año que los hombres, particularmente, en servicios de salud, incluyendo visitas a los consultorios, cuidados de salud y prescripciones (10).

Al medir las relaciones entre las redes sociales y las capacidades de autogerenciamiento en seis países de Europa, se observó que la asociación de capacidades de autogerenciamiento con el apoyo informacional fue especialmente fuerte en el grupo de baja escolaridad, mientras la asociación con apoyo emocional fue más fuerte en el grupo de educación superior. También, el 
hecho que más miembros de la red brinden soporte informativo o soporte emocional, y participen en organizaciones de la comunidad, fue asociado con un mayor automonitoreo (8).

\section{Discusión}

Esta revisión indica que más del 90\% de los artículos fueron escritos en países de altos ingresos. En una proporción similar, utilizaron abordajes cuantitativos en comparación con los cualitativos. En relación con las variables o categorías para analizar o medir las desigualdades, las condiciones étnico/raciales y las condiciones socioeconómicas fueron las más usadas. Dentro de las primeras, aparecen los grupos de minorías étnico/raciales asentados en los países de tiempo atrás, o que vienen llegando como migrantes o refugiados, producto de las crisis económicas, políticas y humanitarias de algunas regiones, especialmente de África y Suramérica. Las segundas incluyen principalmente ingresos, empleo, ocupación, nivel educativo y lugar de residencia.

Otras variables o categorías son menos estudiadas como tema central, como el género, la edad, el tipo de seguro/plan de salud. En cuanto a los aspectos estudiados sobre la diabetes, las investigaciones se centran principalmente en la prevalencia y el riesgo de tener diabetes, el uso y acceso a los servicios de salud, y el autogerenciamiento. En menor proporción se analizaron factores de riesgo asociados.

Los resultados muestran que las desigualdades sociales medidas principalmente por condiciones étnico/raciales y socioeconómicas tienen efectos sobre la incidencia, el tratamiento/manejo y la atención de la diabetes, mientras que, con respecto al género, se evidenció un comportamiento variable entre hombres y mujeres. La condición étnico/racial estuvo relacionada con mayor prevalencia de diabetes, la percepción y vivencia por las minorías étnicas de situaciones de discriminación en los servicios de salud, diferencias en el trato por parte del personal médico, y barreras de comunicación entre pacientes y médicos. También, mayor prevalencia de hábitos de riesgo, asociados con el autocuidado, la alimentación y la actividad física.

Los resultados de estos estudios ofrecen evidencias que refuerzan hallazgos que vienen presentándose en otras investigaciones. Por una parte, la asociación entre desventajas socioeconómicas con la diabetes mellitus tipo 2; y, por otra, un refuerzo entre condiciones socioeconómicas y étnico/raciales. La primera afirmación coincide con las relaciones entre gradiente social y condiciones de salud, es decir, un empeoramiento de las condiciones de salud en la medida que se desciende en la escala socioeconómica (25).

Esto coincide con los resultados que señalan que el bajo nivel educativo, las ocupaciones de menor jerarquía y los ingresos más bajos aumentan el riesgo de tener diabetes, de la misma forma en la que las condiciones de habitación deficitaria, la débil disponibilidad de infraestructuras que incentiven la adopción de estilos de vida saludables y las barreras de acceso a los cuidados de 
salud se transforman en ambientes vulnerables, determinantes para la incidencia de la diabetes $(7,8,33,34,35,36,26,27,28)$.

Más aún, algunos estudios describen de qué forma las condiciones socioeconómicas afectan el cuidado y el tratamiento de la diabetes. La prioridad de llevar el dinero al hogar reduce el tiempo para preparar comidas adecuadas para la enfermedad, por lo que los pacientes optan por comidas preparadas (29), y experimentan dificultades para adquirir la alimentación más adecuada, por ser menos económica y por anteponer a sus hijos a la enfermedad, comprando alimentos básicos para ellos $(30,37,14)$. También, el alto costo de la medicación y la necesidad de más medicamentos prescritos impiden su toma diaria $(37,14)$

Igualmente, la desventaja económica de los pacientes dificulta el control glicémico, debido a la falta de glucómetro en sus casas, además de las dificultades para comprar zapatos apropiados para sus pies, por lo que prefieren comprar ropa para sus hijos $(30,37,14)$. Finalmente, el acceso a especialistas puede ser poco frecuente en las clases con menos recursos económicos, pues depende de su capacidad de pago y del tipo de plan de salud que posean $(30,15)$.

La segunda afirmación, que relaciona las condiciones socioeconómicas y las condiciones étnico/ raciales, combina el gradiente socioeconómico con una dimensión sociocultural que pone en desventaja no solo material a las minorías o ciertos grupos étnicos, sino también, simbólica. Esta doble condición influye en las relaciones sociales y en las prácticas de cuidado, como lo muestran los trabajos con población indígena y con otros grupos minoritarios presentados.

Los resultados presentados coinciden con los de otros estudios que muestran mayor prevalencia de diabetes tipo 2 y menor frecuencia de prácticas de autocuidado en grupos étnicos minoritarios $(27,16,17)$. Frente a la dimensión cultural/simbólica es frecuente encontrar que los pacientes de minorías étnicas perciben tratos discriminatorios y preconcepciones de parte del personal médico. Las diferencias culturales entre los equipos de salud y los pacientes, al igual que las diferencias de idioma, afectan la comunicación y la confianza. Esas diferencias dificultan que los pacientes reciban y acojan las indicaciones para su autocuidado, además limitan su participación en las decisiones sobre su tratamiento $(30,31,32,38,18,19,20)$.

En relación con el género, que es una de las principales categorías para los análisis de las desigualdades sociales relacionadas con los procesos de salud-enfermedad-cuidado, en esta revisión solo hubo dos estudios que mostraron resultados al respecto: una mayor asociación entre nuevos casos de diabetes con el aumento de las desventajas socioeconómicas en el curso de la vida, principalmente en los hombres y mayores gastos de bolsillo y gastos totales para el tratamiento de la diabetes por parte de las mujeres.

Los resultados del estudio de gasto de bolsillo muestran mayores coincidencias con estudios que ofrece la literatura, ya que tienden a mostrar una importante relación entre las desventajas socioeconómicas, el género femenino y, en algunos casos, la pertenencia a grupos étnico/raciales minoritarios. Se describe que las desigualdades en la diabetes son más intensas en mujeres que 
en hombres, debido a que las mujeres de posición socioeconómica desfavorecida concentran una mayor proporción de factores de riesgo — como obesidad, sedentarismo, dieta inadecuada, así como estrés psicosocial— que los hombres que están en las mismas posiciones sociales $(7,21)$.

Otros estudios coincidentes muestran mayor prevalencia de diabetes en mujeres de poblaciones mayoritariamente de origen africano, en comparación con los hombres del mismo país y del mismo origen étnico $(22,23,24)$. Esta relación se evidencia en algunos países de América Latina como Colombia y Argentina $(39,40)$. De la misma forma, se observaron menores controles de hemoglobina glicosilada en las mujeres (41), y mayor índice de masa corporal, clasificado como sobrepeso y obesidad (42).

Sobre el autocuidado, se encontró que el rol de "multicuidadora", impuesto/asumido por las mujeres en la cultura africana, dificultaba el hecho de ser ayudada por su familia para su autocuidado (43). En México se reportó que la actividad física y la alimentación son los dos aspectos más críticos de las mujeres, debido a que en general, los hombres tienen una posición social más favorable, mayor autonomía frente al uso de su tiempo, así como mayor acceso a los recursos monetarios y no monetarios en las familias (44).

\section{Conclusiones}

Al comparar diversos estudios realizados en diferentes latitudes, es posible encontrar evidencias que tienden a confirmar que existe una asociación entre las desigualdades sociales de carácter socioeconómico y cultural y la diabetes, en cuanto a la prevalencia, la incidencia, el tratamiento y el autocuidado. No obstante, para tener evidencia en la región latinoamericana, es necesario intensificar la investigación y la publicación de estudios en el tema, ya que la mayoría de estos, como lo muestra esta revisión, fueron publicados en países de altos ingresos. También, los resultados de las investigaciones evidencian que las condiciones sociales operan de forma acumulativa, al configurar inequidades que afectan las personas, grupos o poblaciones que se encuentran en una mayor vulnerabilidad frente a situaciones de enfermedad y tratamiento, y causan un círculo vicioso entre desventaja/vulnerabilidad social y enfermedad/cuidado.

Para comprender mejor los mecanismos a través de los cuales interactúan las condiciones sociales y de salud, bajo esta forma de círculo vicioso, es necesario desarrollar en mayor medida estudios de tipo cualitativo o mixtos. Estos tipos de abordaje permiten profundizar en las trayectorias de vida de las personas para explorar con mayor nivel de detalle — siguiendo casos particulares, en medio de contextos específicos - las formas concretas de estas interacciones reciprocas entre las desigualdades sociales y las desigualdades en salud, como dos caras de la misma moneda. Así, entonces, los resultados situados contextualmente de este tipo de investigaciones ofrecerán insumos para desarrollar estrategias locales y singulares para prevenir, mitigar daños, fortalecer el automanejo, mejorar el acceso, la oferta y el uso de los servicios, sin que ello implique renunciar a medidas globales. 


\section{Agradecimientos}

Agradecimientos a la profesora Rita de Cássia Lima Duarte, de la Universidad Federal de Espírito Santo (UFES), por la revisión y sus aportes para la escritura de este artículo.

\section{Referencias}

1. Organización Mundial de la Salud. Global Report on Diabetes. Library Cataloguing-in-Publication Data. Washington: OMS; 2016.

2. Organización Mundial de la Salud. Global Health Estimates: Deaths be Cause, Age, Sex and Country, 2000-2012. Ginebra: OMS; 2014.

3. Chen L, Magliano DJ, Zimmet PZ. The worldwide epidemiology of type 2 diabetes mellitus-present and future perspectives. Nat Rev Endocrinol. 2012;8:228-236.

4. Domínguez E. Desigualdades sociais e diabetes mellitus. Revista Cub Endocr. 2013;24(2):200-213.

5. Brown AF, Ettner SL, Piette J, Weinberger M, Gregg E. Socioeconomic position and health among persons with diabetes mellitus: a conceptual framework and review of the literature. Epidemiol Rev. 2004;26:63-77.

6. Agardh E, Ahlbom A, Andersson T, Efendic S, Grill V, Hallqvist J, et al. Explanations of socioeconomic differences in excess risk of type 2 diabetes in Swedish men and women. Diabetes Care. 2004;27:716-721.

7. Tang M, Chen E, Krewski D. Gender-related differences in the association between socioeconomic status and self-reported diabetes. Int J Epidemiol. 2003;32:381-385.

8. Santana P, Costa C, Loureiro A, Raposo J, Boavida J. Geografias da Diabetes Mellitus em Portugal: Como as Condições do Contexto Influenciam o Risco de Morrer. Acta Med Port. 2014 mayjun;27(3):309-317.

9. Saedah S, Imperatore G, Beckles G. Socioeconomic status and mortality. Contribution of health care access and psychological distress among U.S. adults with diagnosed diabetes. Diabetes Care. 2013;36:49-55.

10. Saedah S, Lochner K. Socioeconomic status and risk of diabetes-related mortality in the U.S. Public Health Rep. 2010 may-jun;125(3):377-388.

11. Connolle V, Unwin N, Sherriff P, Bilous R, Kelle W. Diabetes prevalence and socioeconomic status: a population based stude showing increased prevalence of type $2 \mathrm{DM}$ in deprived areas. J Epidemiol Communite Health. 2000;54:173-177.

12. Mendes KS, Silveira RC, Galvao CM. Revisão integrativa: método de pesquisa para a incorporação de evidências na saúde e na enfermagem. Enferm. Florianópolis. 2008 dic.; 17(4):758-764.

13. Moher D, Liberati A, Tetzlaff J, Altman DG. The PRISMA Group. Preferred Reporting Items for Systematic Reviews and Meta-Analyses: The PRISMA Statement. PLoS Med. 2009 jul.; 6(7):e1000097. 
14. Nagelkerk J, Reick K, Meengs L. Perceived barriers and effective strategies to diabetes selfmanagement. J Adv Nurs. 2006;54:151-158.

15. Peek ME, Odoms-Eoung A, Quinn MT, Gorawara-Bhat R, Wilson S, Chin MH. Race and shared decision-making: perspectives of African-Americans with diabetes. Soc Sci Med. 2010 jul.;71(1):1-9.

16. Johnson M, Baird W, Goeder E. Understanding issues involved in the transfer of diabetes care to general practice: the patient perspective. Qual Prim Care. 2006;14:247-252.

17. Caban A, Walker EA, Sanchez S, Mera MS. "It Feels Like Home When Eat Rice and Beans": Perspectives of Urban Latinos Living With Diabetes. Diabetes Spectr. 2008;21:120-127.

18. Hernández A. Las desigualdades sociales en diabetes mellitus 2, hipertensión arterial y obesidad en las poblaciones afrohondureña y mestiza de La Ceiba. Honduras, 2013 [Tesis para obtener el Grado de Doctor en Salud Pública]. [Guatemala]: Universidad de San Carlos de Guatemala; 2013.

19. Soler J, Marsal JR, Serna C, Real J, Cruz I, López A. La población inmigrante controla peor su diabetes que la población autóctona. Gac Sanit. 2013;27(1):19-25.

20. Torres C, Barceló A. Injusticia social y necesidades no cubiertas: mujeres y diabetes en las Américas. Diabetes Voice. 2009;54:12-17.

21. Shai I, Jiang R, Manson J. Ethnicity, obesity, and risk of type 2 diabetes in women. Diabetes Care. 2006;29:1585-1589.

22. Chen R, Cheadle A, Johnson D, Duran, B. U.S trends in receipt of appropriate diabetes clinical and self-care from 2001 to 2010 and racial/ethnic disparities in care. Diabetes Educ. 2014 novdic;40(6):756-766.

23. Detz A, Mangione CM, Nunez de Jaimes F, Noguera C, Morales LS, Tseng CH, et al. Language concordance, interpersonal care, and diabetes self-care in rural Latino patients. J Gen Intern Med. 2014 dic;29(12):1650-1656.

24. Organización Panamericana de la Salud. Best practices in gender, ethnicity and health. Washington: OPS; 2009.

25. Mújica OJ. Cuatro cuestiones axiológicas de la epidemiología social para el monitoreo de la desigualdad en salud. Rev Panam Salud Publica. 2015;38(6):433-441.

26. Agardh E, Allebeck P, Hallqvist J, Moradi T, Sidorchuk A. Type 2 diabetes incidence and socio-economic position: a systematic review and meta-analysis. International Journal Epidemiol. 2011;40:804-818.

27. Schmitz N, Nitka D, Gariepe G, Malla A, Wang J, Boeer R, et al. Association between neighborhoodlevel deprivation and disability in a community sample of people with diabetes. Diabetes Care. 2009;32(11):1998-2004.

28. Daria S. Análisis de las desigualdades económicas en la Prevalencia de la diabetes y la obesidad en Canarias. Rev Enf. 2008;4:51-60.

29. Organización Panamericana de la Salud. Health in the Americas. Washington: OPS; 2007. 
30. Ramal E, Petersen AB, Ingram KM, Champlin AM. Factors that influence diabetes self-management in Hispanics living in low socioeconomic neighborhoods in San Bernardino, California. J Immigr Minor Health. 2012 dic.;14(6):1090-1096.

31. Sanders MP. Diabetes and the disadvantaged: reducing health inequalities in the UK. Londres: All Party Parliamentary Group for Diabetes; 2006.

32. Pickett KE, Kelle S, Brunner E, Lobstein T, Wilkinson RG. Wider income gaps, wider waistbands? An ecological study of obesity and income inequality. J Epidemiol Communite Health. 2005;59:670-674.

33. Wang Y, Chuang L, Bateman W. Focus Group Study Assessing Self-Management Skills of Chinese Americans with Type 2 Diabetes Mellitus. J Immigrant Minority Health. 2012;14:869-874.

34. Wester GP, Schellevis FG, Bakke DH, Groenewegen PP, Bensing JM, Van Der Zee J. Monitoring health inequalities through general practice: the Second Dutch National Survey of General Practice. Eur. J. Public Health. 2005;15(1):59-65.

35. Weng C, Coppinit D, Sönksen P. Geographic and social factors are related to increased morbidity and mortality rates in diabetic patients. Diabet Med. 2000;17:612-617.

36. Lundberg PC, Thrakul SJ. Type 2 diabetes: how do Thai Buddhist people with diabetes practice selfmanagement? Adv Nurs. 2012 mar.;68(3):550-558.

37. Adams CR. Lessons learned from urban Latinas with type 2 diabetes mellitus. J Transcult Nurs. 2003; 14:255-265.

38. Pilkington FB, Daisuki I, Breant T, Dinca-Panaitescu M, Dinca-Panaitescu S, Raphael D. The experience of living with diabetes for low-income Canadians. Can J Diabetes. 2010;34:119-126.

39. Marro MJ, Moreira A, Da Costa I. Desigualdades regionales en la mortalidad por diabetes mellitus y en el acceso a la salud en Argentina. Cad. Saúde Pública. 2017;33(9):e00113016.

40. Cerezo-Correa MDP, Cifuentes-Aguirre OL, Nieto-Murillo E, Parra-Sánchez JH. Desigualdades de la morbilidad por enfermedades crónicas según determinantes estructurales e intermediarios. Rev. Gerenc. Polit. Salud. 2012; jul-dic.;11(23):165-188.

41. Bacigalupe A, Esnaola S, Fraile I, Ibarra J, Urraca J, Sánchez S, et al. Desigualdades sociales en la atención a la diabetes tipo 2 en la Comarca Araba. Vitoria: Departamento de Salud. Gobierno Vasco; 2017.

42. Rossaneis MA, Haddad MDC, Mathias L, Marcon SS. Diferenças entre mulheres e homens diabéticos no autocuidado com os pés e estilo de vida. Rev. Latino-Am. Enfermagem 2016;24:e2761.

43. Carter-Edwards L, Skelle AH, Cagle CS, Appel SJ. Thee care but don't understand: family support of African American women with type 2 diabetes. Diabet Educt. 2004;30:493-501.

44. Trujillo LE, Nazar A. Autocuidado de diabetes: una mirada con perspectiva de género. Est Demogr Urbanos [Internet]. 2011;26(3). Disponible en: http://www.scielo.org.mx/scielo.php?script=sci_artt ext\&pid=S0186-72102011000300639\&lng=es\&nrm=iso

Notas

* Artículo de revisión. Este documento presenta parte de los resultados de la tesis para optar al título de Doctor en Salud Colectiva "Desigualdades sociais em experiências de adoecimento por diabetes mellitus tipo 2", en la Universidade Federal do Espírito Santo, UFES 2016-2019 financiado con recursos de CAPES (Coordenação de Aperfeiçoamento de Pessoal de Nível Superior). 